\title{
Conductas de salud y factores de riesgo en la salud sexual y reproductiva de una población universitaria
}

\author{
Sonia Acosta ${ }^{1}$, Edgar Ibáñez ${ }^{2}$, Andrea Alfonso ${ }^{3}$, Linda Cifuentes ${ }^{3}$, \\ Sandra Gamba ${ }^{3}$, Carolina Mojica ${ }^{3}$, Vilma Vargas ${ }^{3}$, Efraín Patiño 4
}

1. Enfermera, Especialista en Perinatología. Profesora. Facultad de Enfermería, Universidad El Bosque. 2. Ingeniero, Especialista en Estadística, Especialista en Docencia Universitaria, Candidato a Maestría en Epidemiología Clínica, Instructor Asociado. Facultad de Enfermería, Universidad El Bosque.

3. Enfermeras, Universidad El Bosque.

4. Ingeniero Sistemas. Profesor Asistente. Facultad de Ingeniería de sistemas Universidad El Bosque.

Correspondencia: edgant11@yahoo.com

Recibido: 05-04-2010 / Aceptado: 06-01-2010

\begin{abstract}
Resumen
Este estudio, iniciado en el año 2008, tuvo como objetivo describir los factores de riesgo y las conductas de la salud sexual y reproductiva de una población universitaria. Se realizó un estudio observacional de corte transversal, con una muestra conformada por 49 I estudiantes de una universidad de Bogotá. Los resultados muestran que el género predominante fue el femenino $(63,1 \%$,), con una edad promedio de 20,4 2,6 años; el $80 \%$ inició su actividad sexual y la edad entre los 15 y los 18 años. El método anticonceptivo más usado es el preservativo y el 37,9\% tiene conocimientos escasos sobre anticoncepción. La prevalencia de infecciones de transmisión sexual es baja, pero sólo la mitad han recibido tratamiento. El I0\% de la población se ha realizado la prueba para el virus de inmunodeficiencia humana. El I $0 \%$ de la población ha presentado embarazos, de los cuales el 75,3\% no han sido planeados y el $47,4 \%$ han terminado en aborto, de los cuales el $80 \%$ han sido inducidos.

Hay cifras altas de antecedentes familiares de cáncer. El $84 \%$ de los hombres no se realizan el auto examen de testículo. En las mujeres, el 56\% se realiza auto examen de seno. La prevalencia de citologías es baja (38\%), no todas han reclamado el resultado y aquellas que presentaron anormalidades (78\%) no recibieron tratamiento. El 86,9\% de los estudiantes desean que la universidad desarrolle el programa de salud sexual y reproductiva; el medio preferido es la consulta personalizada y la pagina web con asesoría en línea. El análisis de los resultados permite anotar que los jóvenes requieren programas de promoción de la salud que conlleven a que éstos asuman su sexualidad con autonomía, responsabilidad e información adecuada, en el marco de sus derechos sexuales y reproductivos.

Palabras clave: anticoncepción, educación sexual, enfermedades de transmisión sexual, salud sexual y reproductiva.
\end{abstract}




\section{Abstract \\ Health behaviors and risk factors in sexual and reproductive health of a university population}

This study, conducted in 2008, aimed to describe risk factors and behaviors of sexual and reproductive health in a university population. We conducted a cross-sectional observational study with a sample comprised of 49I students from a university in Bogota. The results show that females were predominant (63.1\%), with an average age of $20.4 \pm 2.6$ years, $80 \%$ began their sexual activity between 15 and 18 years of age. The most widely used contraceptive method is the condom and $37.9 \%$ have poor knowledge about contraception. The prevalence of sexually transmitted infections is low, but only half have received treatment. $10 \%$ of the population has made testing for human immunodeficiency virus. $10 \%$ of the population has presented pregnancies, out of which $75.3 \%$ have not been planned and 47.4\% have ended in abortion, of which $80 \%$ were induced.

There are high numbers of family cancer history. 84\% of men do not perform a testicular selfexamination. $56 \%$ of women perform breast self-exam. The prevalence of cytology is low (38\%), not all women have claimed their outcome and those with abnormalities (78\%) received no treatment. 86.9\% of students want the university to develop the program of sexual and reproductive health, the preferred medium is consultation and personalized website with online counseling. The analysis of the results allows noting that youth require programs to promote health that lead them to assume their sexuality with autonomy, responsibility, and appropriate information as part of their sexual and reproductive rights. Keywords: contraception, sex education, sexually transmitted diseases, sexual and reproductive health.

\section{Introducción}

La sexualidad y la reproducción están íntimamente ligadas a la calidad de vida, tanto en el ámbito de lo individual como de lo social. La salud sexual y reproductiva (SSR) se refiere a un estado general de bienestar físico, mental y social, no a la mera ausencia de enfermedades o dolencias en todos los aspectos relacionados con la sexualidad y la reproducción. Lo anterior conlleva a ejercer los derechos sexuales y reproductivos (DSR) (1). Mejorar la SSR de las poblaciones es una meta de los gobiernos y de los organismos de salud como se ve reflejado en los objetivos del milenio y en las políticas nacionales y distritales, lo cual requiere el concurso de todos los entes para impactar en los ámbitos personal, familiar, social y comunitario a lo largo de todo el ciclo vital (2-4).

En la Conferencia Mundial de Población y Desarrollo celebrada en el Cairo en 1994, se aprobaron políticas dirigidas a garantizar los derechos a la SSR; además se recomendó como objetivo abordar estos temas con los jóvenes, en particular, los embarazos no deseados, el aborto y las infecciones de transmisión sexual (ITS), mediante el fomento de actitudes responsables y la prestación de servicios apropiados con orientaciones adecuadas para esa edad (2). Cabe resaltar que más de la mitad de los habitantes del mundo son menores de 25 años, de ellos un tercio tiene entre 10 y 24 años y un $80 \%$ de éstos viven en países en vía desarrollo (5); estas cifras se duplicaron en algunos continentes entre 1960 -1980 y se espera un incremento en la población joven en el 2020, la cual superará los 1200 millones de habitantes (6).

La sociedad debe asumir responsablemente la obligación de ofrecer a ese grupo poblacional programas de SSR que se adapten a sus necesidades, es 
por esto que se hace urgente realizar investigaciones, diagnósticos e intervenciones en el grupo de jóvenes. En Colombia, desde 1985 se observa un incremento de la fecundidad de las mujeres entre 15-19 ańos; el $7,6 \%$ son sexualmente activas y utilizan un método moderno de anticoncepción; una de cada 3 , ha tenido relaciones sexuales, muchas veces sin protección, de manera que se incrementa el riesgo del embarazo no deseado, la adquisición de infecciones de transmisión sexual, VIH/SIDA y la morbi-mortalidad por aborto inseguro; este último es la tercera causa de muerte materna en adolescentes. El 15\% de las adolescentes han sido madres y el $4 \%$ a esta edad están embarazadas de su primer hijo (7-9).

A pesar que en la conferencia de El Cairo (1994), la comunidad internacional reconoció oficialmente, y por primera vez, que la SSR presenta necesidades diferentes durante la fase juvenil a las experimentadas por los adultos, el problema sigue vigente. La juventud es un segmento poblacional con características propias y por lo tanto, entre sus derechos y necesidades, está el acceso a servicios y programas de salud diferenciados y especializados. En esta conferencia se determinó que era un gran reto para los países en vías de desarrollo, la aplicación de un programa de acción dirigido al apoyo de la juventud, puesto que su implementación requiere de una construcción social que debe resolver las perspectivas no equitativas de género y las condiciones de pobreza, entre otros, $(10,11)$.

De otro lado, la población de jóvenes es un grupo con problemas de SSR que requieren ser abordados, pues la mayoría de ellos inician su actividad sexual a edades muy tempranas y no tienen una información apropiada y oportuna en temas de sexualidad y salud reproductiva, esto conlleva a que no tengan claro sus derechos y responsabilidades en este aspecto (12). Un espacio importante para desarrollar programas de promoción y prevención en SSR los colegios y las universidades (13), comporta como institución mediadora entre la dimensión social y la individual garantiza la educación integral de los educandos y por tanto, En ese sentido es imposible ignorar el papel fundamental que debe desempeñar en la educación sobre salud sexual y reproductiva estas instituciones (14), considerando a los jóvenes como un recurso muy valioso para el progreso social, económico y político del país, que deben asumir su sexualidad con autonomía, responsabilidad e información adecuada, en el marco de sus derechos sexuales y reproductivos (15).

En esta investigación se describe los factores de riesgo y las conductas de SSR de una población universitaria, con el fin de orientar el diagnóstico e intervención a grupos que comparten características similares. Es de resaltar que el profesional de enfermería tiene un papel protagónico en el desarrollo de estrategias, ya que en ellos recae gran parte de las acciones específicas de promoción de salud y de prevención de la enfermedad que conlleven a mejorar la SSR de las poblaciones, con el objetivo de lograr en las personas una sexualidad sana y libre de riesgos.

\section{Materiales y métodos}

Este estudio se clasifica como observacional de corte transversal. La población objeto de estudio fueron 3966 estudiantes universitarios matriculados en el año 2008; el tipo de muestreo estratificado por facultad por afijación proporcional, con una precisión absoluta del $5 \%$, una potencia del $80 \%$ y un nivel de confianza del $95 \%$, siendo la muestra final de 491 estudiantes. Las variables socios demográficas involucradas en el estudio fueron: edad, género, estado civil y variables relacionadas con los temas de actividad sexual, anticoncepción, infecciones de transmisión sexual, gestaciones, prevención de cáncer, asesoría sobre SSR.

Las variables cualitativas se describieron mediante distribuciones de frecuencias y porcentajes; las variables cuantitativas con medidas de tendencia central como el promedio y medidas de dispersión como la desviación estándar, se determinó la variación mediante el coeficiente de variación u homogeneidad (0-10\% homogéneo, 11-20\% medianamente homogéneo, mayor del $20 \%$ heterogéneo). Los errores de muestreo relativo se realizaron para las categorías más prevalentes de las variables y para aquellas en donde la respuesta era si o no solamente se tomó el sí, se consideraron buenas inferencias aquellos en donde los errores de muestreo fueron inferiores al $30 \%$. Se digitó y depuró en Excel versión 2003 y se proceso la información para muestras complejas en el paquete SPSS versión 15. 
Tabla 1. Características generales de la población estudiada.

\begin{tabular}{|c|c|c|c|c|c|c|c|}
\hline & Categoría & Población & Muestra & Porcentaje & & $5 \%$ & error muestral \\
\hline & & Estudiantes & Estudiantes & (\%) & Inf. & Sup. & relativo(\%) \\
\hline Inicio actividad & Si & 3187 & 393 & 80,4 & 76,4 & 83,8 & 2,3 \\
\hline sexual & No & 779 & 98 & 19,6 & 16,2 & 23,6 & - \\
\hline & Menos o igual a 14 años & 460 & 54 & 14,6 & $\mathrm{II}, 2$ & 18,7 & 13,3 \\
\hline actividad sexual & Entre 15 y 18 años & 2130 & 264 & 67,5 & 62,4 & 72,2 & 4,4 \\
\hline & Mayor de 18 años & 568 & 72 & 18,0 & 14,3 & 22,3 & II,6 \\
\hline & I & 1940 & 243 & 61,8 & 56,7 & 66,7 & 4,8 \\
\hline $\begin{array}{l}\text { Compañeros (as) } \\
\text { Sexuales }\end{array}$ & 2 a 4 & 1018 & 124 & 32,4 & 27,8 & 37,5 & 8,1 \\
\hline & Más de 4 & $|8|$ & 22 & 5,8 & 3,8 & 8,7 & 21,4 \\
\hline Compañero (a) & Si & 2012 & 258 & 64,3 & 59,3 & 69,1 & 4,5 \\
\hline Sexual Actual & No & 1116 & 128 & 35,7 & 30,9 & 40,7 & 7,5 \\
\hline Compañero (a) & Permanente & 1440 & 189 & 72,6 & 66,4 & 78,0 & 6 \\
\hline Sexual & Ocasional & 545 & 66 & 27,4 & 22,0 & 33,6 & 11,8 \\
\hline Frecuencia de & $\begin{array}{l}\text { Menos de I relación por } \\
\text { semana } \\
\text { De I a } 3 \text { relaciones por }\end{array}$ & 878 & II4 & 44,1 & 38,0 & 50,4 & 8,3 \\
\hline actividad sexual & $\begin{array}{l}\text { semana } \\
\text { Más de } 3 \text { relaciones por }\end{array}$ & 910 & 115 & 45,7 & 39,4 & 52,0 & 8,6 \\
\hline & semana & 204 & 27 & 10,2 & 7,1 & 14,6 & 18,8 \\
\hline
\end{tabular}

\section{Resultados}

\section{Características generales}

El género predominante, dentro de la población estudiada, fue el femenino, con un $63.1 \%(n=310$, $\mathrm{N}=2284)$ y el masculino fue de $36.9 \%(\mathrm{n}=181$, $\mathrm{N}=1682$ ). La edad promedio fue de $20.4 \pm 2.6$; el $95 \%$ de la población se encuentra entre 15.2 años y 25.6 años, con un mínimo de edad de 16 años y un máximo de 40 años, la variabilidad es medianamente homogénea $(\mathrm{CV}=12.9 \%)$. Con respecto al estado civil, predominan los solteros, que representaron un $93.7 \%(n=460)$ seguido de unión libre con un $4.1 \%(n=20)$ y con baja frecuencia casados con un $1.6 \%(n=8)$, separados con $0.4 \%(n=2)$ y viudo con un $0.2 \%(n=1)$.

\section{Actividad sexual}

El 80,4\% anota tener actividad sexual (hombres el $89 \%$ y mujeres el $75 \%$ ), siendo la edad de inicio entre 15 a 18 ańos (sin diferencia de género) con 1 compańero sexual, seguido de 2 a 4, compañeros; el $64,3 \%$ tiene compañero actualmente, siendo más frecuente el compañero permanente. Con respecto a la actividad sexual, la frecuencia fue de 1 a 3 relaciones por semana, datos similares se obtuvieron para menos de una relación por semana. Estos datos se muestran en la Tabla 1.

\section{Anticoncepción}

Un $82,1 \%$ de los estudiantes está utilizando algún método de anticoncepción, siendo el más utilizado el preservativo, seguido por los anticonceptivos orales. El preservativo es utilizado en todas las relaciones solo por la mitad de la población, siendo frecuente su uso en la relación vaginal seguida por el uso en todos los tipos de relación. La decisión en la elección del método, el 75,5\% manifiesta haberla tomado por iniciativa propia, el $62,3 \%$ no recibe control del método anticonceptivo. La razón más frecuente por la que se elige el método de anticoncepción es por la facilidad de uso y consecución. El preservativo es considerado como el mejor método seguido de los anticonceptivos inyectables. El 57.5\% de la población considera sus conocimientos sobre anticoncepción como muy buenos, Tabla 2 . 
Tabla 2. Métodos de anticoncepción.

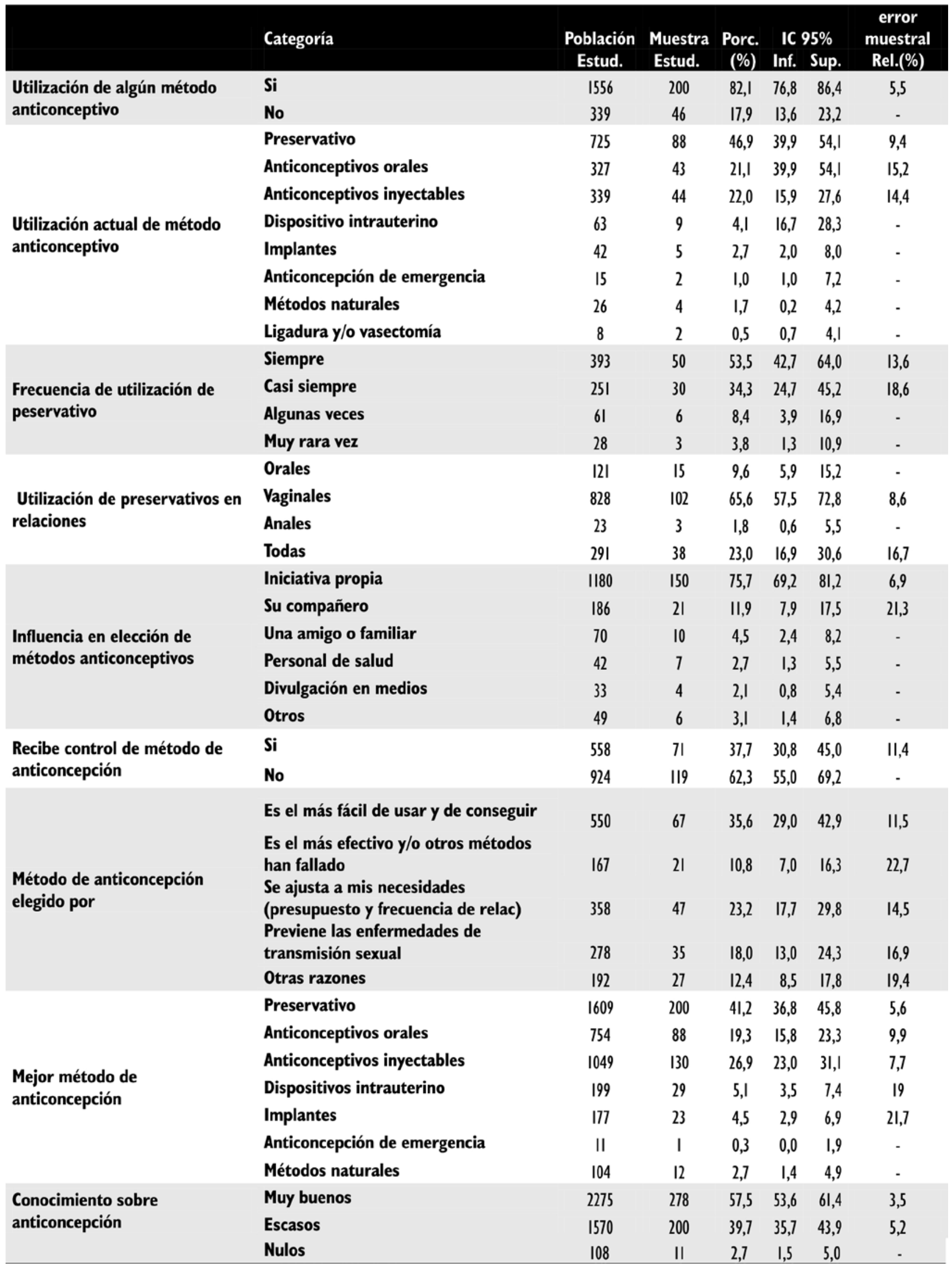


Tabla 3. Infecciones de transmisión sexual.

\begin{tabular}{|c|c|c|c|c|c|c|c|}
\hline & \multirow[t]{2}{*}{ Categoría } & \multirow{2}{*}{$\begin{array}{l}\text { Población } \\
\text { Estudiantes }\end{array}$} & \multirow{2}{*}{$\begin{array}{c}\text { Muestra } \\
\text { Estudiantes }\end{array}$} & \multirow{2}{*}{$\begin{array}{c}\text { Porcentaje } \\
(\%)\end{array}$} & \multicolumn{2}{|c|}{ IC $95 \%$} & \multirow{2}{*}{$\begin{array}{l}\text { error muestral } \\
\text { relativo(\%) }\end{array}$} \\
\hline & & & & & Inf. & Sup. & \\
\hline \multirow{2}{*}{$\begin{array}{l}\text { Conocimiento sobre infecciones de } \\
\text { transmisión sexual }\end{array}$} & Si & 3804 & 468 & 96,0 & 93,9 & 97,4 & 0,9 \\
\hline & No & 157 & 22 & 4,0 & 2,6 & 6,1 & - \\
\hline \multirow{2}{*}{$\begin{array}{l}\text { Presencia de infecciones de transmisión } \\
\text { sexual }\end{array}$} & $\mathrm{Si}$ & 164 & 21 & 4,3 & 2,8 & 6,5 & 6,5 \\
\hline & No & 3654 & 448 & 95,7 & 93,5 & 97,2 & - \\
\hline \multirow{2}{*}{$\begin{array}{l}\text { Tratamiento a la infección de transmisión } \\
\text { sexual }\end{array}$} & Si & 84 & 11 & 49,2 & 29,5 & 69,2 & 69,2 \\
\hline & No & 86 & 11 & 50,8 & 30,8 & 70,5 & - \\
\hline \multirow{2}{*}{$\begin{array}{l}\text { Realización de Tatuajes y/o Piercing, } \\
\text { utilización de Jeringas con otras personas }\end{array}$} & $\mathrm{Si}$ & 911 & 117 & 24,1 & 20,4 & 28,3 & 28,3 \\
\hline & No & 2867 & 351 & 75,9 & 71,7 & 79,6 & - \\
\hline \multirow{2}{*}{ Realización de prueba de VIH } & Si & 392 & 55 & 10,5 & 8,1 & 13,4 & 13,4 \\
\hline & No & 3356 & 408 & 89,5 & 86,6 & 91,9 & - \\
\hline \multirow{2}{*}{ Asesoría Pre y Post de la prueba del VIH } & Si & 247 & 35 & 62,9 & 49,4 & 74,7 & 74,7 \\
\hline & No & 146 & 20 & 37,1 & 25,3 & 50,6 & - \\
\hline
\end{tabular}

Tabla 4. Gestaciones.

\begin{tabular}{|c|c|c|c|c|c|c|c|}
\hline & \multirow{2}{*}{ Categoría } & \multirow{2}{*}{$\begin{array}{l}\text { Población } \\
\text { Estudiantes }\end{array}$} & \multirow{2}{*}{$\begin{array}{c}\text { Muestra } \\
\text { Estudiantes }\end{array}$} & \multirow{2}{*}{$\begin{array}{c}\text { Porcentaje } \\
(\%)\end{array}$} & \multicolumn{2}{|l|}{ IC $95 \%$} & \multirow{2}{*}{$\begin{array}{l}\text { error muestra } \\
\text { relativo(\%) }\end{array}$} \\
\hline & & & & & Inf. & Sup. & \\
\hline \multirow{2}{*}{$\begin{array}{l}\text { Ha estado o está embarazada o en el caso de } \\
\text { ser hombre su compañera? }\end{array}$} & $\mathrm{Si}$ & 341 & 47 & 10,0 & 7,6 & 13,1 & 13,9 \\
\hline & No & 3057 & 372 & 90,0 & 86,9 & 92,4 & - \\
\hline \multirow{2}{*}{ Embarazo planeado } & $\mathrm{Si}$ & 74 & 12 & 24,7 & 14,3 & 34,2 & 29,2 \\
\hline & No & 226 & 31 & 75,3 & 60,8 & 85,7 & - \\
\hline \multirow{2}{*}{ Presentación de Abortos } & $\mathrm{Si}$ & 136 & 21 & 47,4 & 32,4 & 62,3 & 20,9 \\
\hline & No & 151 & 20 & 52,6 & 37,7 & 67,1 & - \\
\hline \multirow{2}{*}{ Tipo de aborto } & Espontáneo & 32 & 6 & 19,8 & 9,2 & 37,4 & 38,3 \\
\hline & Inducido & 136 & 18 & 80,2 & 62,6 & 90,8 & 22,8 \\
\hline
\end{tabular}

\section{Infecciones de transmisión sexual}

La gran mayoría manifiesta tener conocimientos sobre ITS. Una cuarta parte de la población ha tenido tatuajes y/o piercing o ha compartido jeringas con otras personas. Un porcentaje muy bajo (10,5\%) se han practicado la prueba de VIH, donde el 37,1\% no han recibido asesoría pre ni post-prueba, Tabla 3.

\section{Gestaciones}

Los embarazos se han presentado en el $10 \%$ de la población, de los cuales el $75.3 \%$ no han sido planeados, y el $47,4 \%$ de las gestaciones han terminado en aborto, donde el $80 \%$ han sido inducidos, Tabla 4.

\section{Prevención del cáncer en los hombres}

El 48,1\% de la población tiene antecedentes familiares de cáncer, muy pocos saben sobre el auto examen de testículo y el $84,1 \%$ de la población no se realiza el auto examen y por lo general no se lo practican con regularidad, Tabla 5.

\section{Prevención del cáncer en mujeres}

En las mujeres, un alto porcentaje tiene antecedentes familiares de cáncer y el 56\% se realizan el auto examen de seno, lo hacen una vez al mes, pero en cualquier momento del ciclo. La edad de la primera menstruación que predomina es la de 10 a 13 ańos, la frecuencia de realización de la citología es baja y quienes se la hacen lo hacen anualmente; no todas las mujeres han conocido el resultado de su citología, Tabla 6.

\section{Asesoría sobre SSR}

Un alto porcentaje de los estudiantes ven la necesidad de crear un programa de SSR en la 
Tabla 5. Prevención del cáncer en hombres.

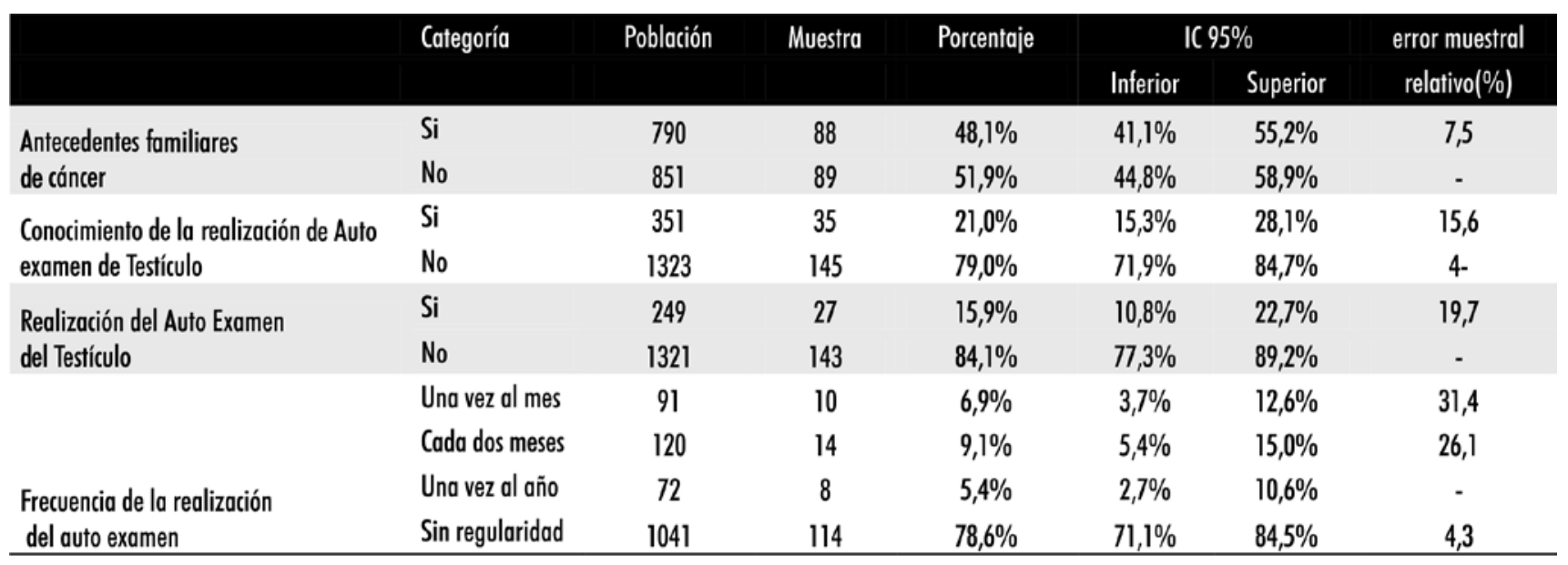

Tabla 6. Prevención del cáncer en mujeres.

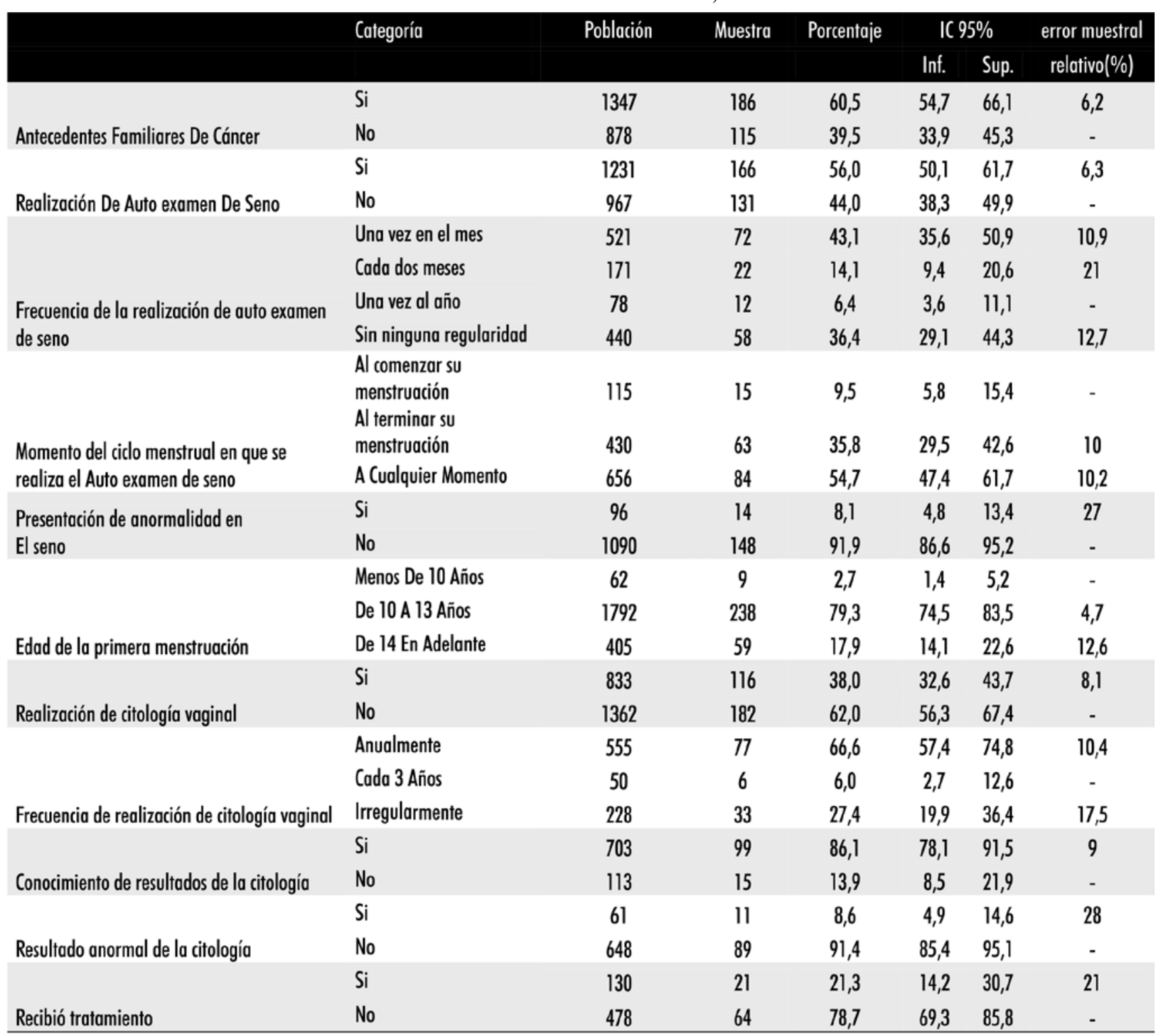


Tabla 7. Programa de salud sexual y reproductiva.

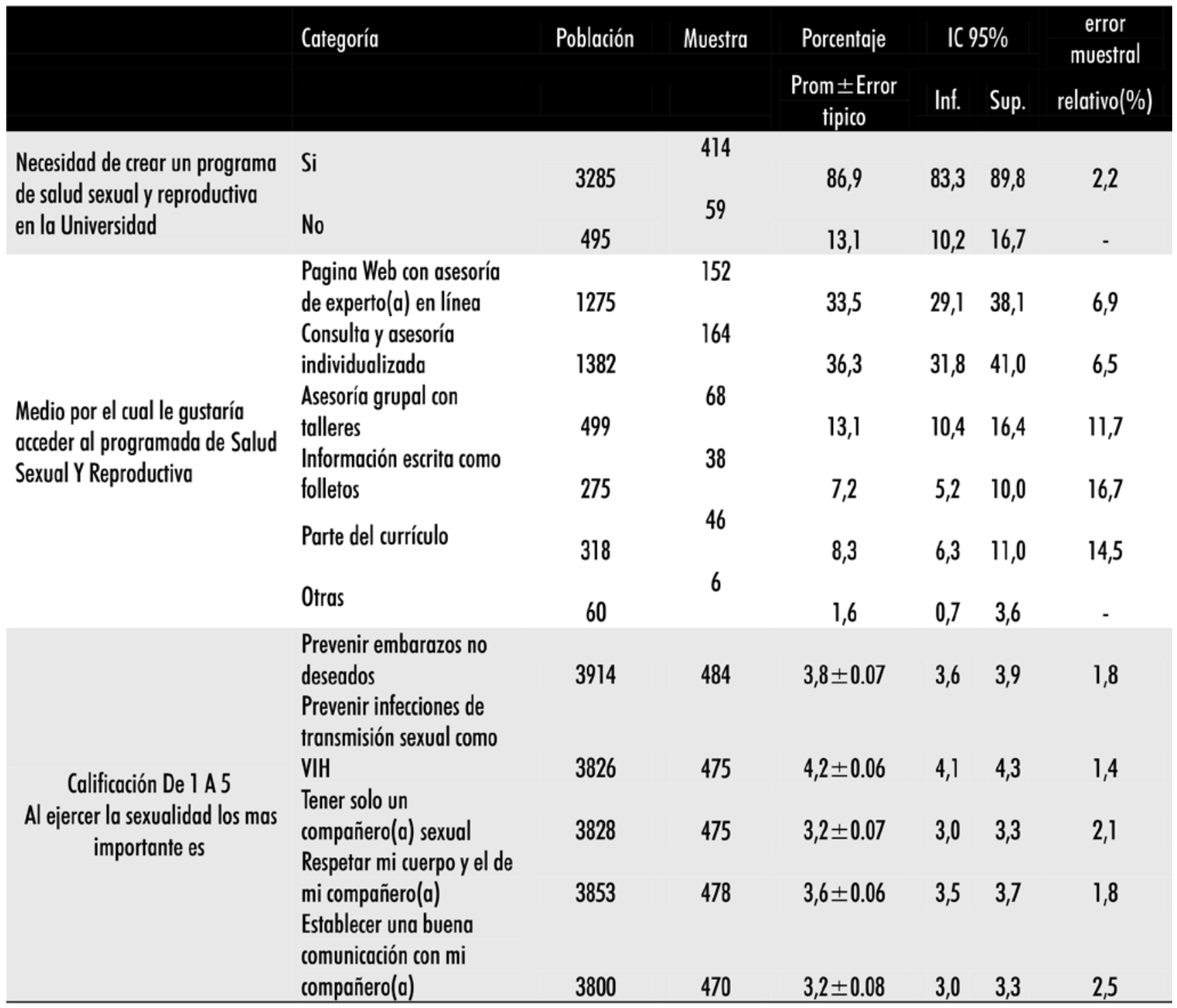

universidad, a través de la consulta individualizada, seguido de la página la web, con asesoría de un (a) experto (a) en línea. Al ejercer la sexualidad se considera que es muy importante la prevención de infecciones de transmisión sexual, seguida de la prevención de embarazos, Tabla 7.

\section{Discusión}

La organización mundial de la salud (OMS) define la adolescencia como la etapa de la vida de los individuos comprendida entre los 10 y los 19 ańos de edad, mientras que la juventud es la etapa comprendida entre los 15 y 24 ańos (23). En este estudio el $95 \%$ de los estudiantes se encuentran en este rango de edad y el $25 \%$ son adolescentes, con un predominio de mujeres. El $80 \%$ de la población investigada informa haber iniciado su actividad sexual, datos similares a los reportados en los estudiantes americanos donde se encontró un $40-90 \%$ y en los estudiantes chilenos el 78\% de los hombres y el $41 \%$ de las mujeres eran activos sexualmente $(13,18)$. Esta cifra es alta si se tienen en cuenta que las relaciones sexuales prematrimoniales en comunidades universitarias de otros países como China presentan $6.1 \%$ de los hombres y el $4.3 \%$ de las mujeres, en Nueva Zelanda el 8.5\%, en Sudáfrica el 17\%, en Australia el 28\%, y en Cuba el 51\% (17).

El inicio de la actividad sexual en el 14,6\% de la población estudiada fue antes de los 14 años. Este es un porcentaje alto comparado con otros estudios 
que reportan un $5.6 \%$ en Cuba (14), España $10 \%$ (16), y en Colombia según la encuesta nacional de demografía y salud (ENDS), un 11\% de las mujeres iniciaron su vida sexual antes de los 15 años (8). La edad de inicio de la actividad sexual ha ido disminuyendo como reportan estudios realizados en El Salvador que encontró que para 1998 la edad promedio a la primera relación coital para las adolescentes entre 15 y 24 años fue de 16.4 años, 15.3 años para las menores de 20 ańos y 17.0 para las de 20 a 24 ańos. Esto evidencia que las relaciones sexuales se inician a más temprana edad (19).

En cuanto al número de compañeros sexuales, se encontró que un 32,4\% reportó 2 a 4 y el $61.8 \%$ un sólo compañero. Cifra similar a lo encontrado en la Universidad del Rosario, donde un 74\% manifiestan haber tenido menos de tres parejas sexuales desde el inicio de la vida sexual activa (9). De acuerdo al informe del Centro para el Control de Enfermedades de Atlanta, hasta la tercera parte de los adolescentes norteamericanos encuestados han tenido seis o más parejas sexuales desde el inicio de su sexualidad (13).

En cuanto a la anticoncepción, el $82 \%$ de la población utiliza algún método anticonceptivo, cifra similar a la encontrada en la Universidad del Rosario que lo reporta en un $88 \%$. Estos datos no cubren el uso correcto y oportuno del método. La ENDS encontró que el $77 \%$ de las mujeres en unión de pareja y el $84 \%$ de las que las que no lo están y son sexualmente activas han empleado al menos un método anticonceptivo (8). El 46.9\% de la población encuestada dice haber utilizado el preservativo, cifra baja comparada con otros estudios como en Cuba que es utilizado por el 61.9\%, en España por el 95\% de los hombres y el $92 \%$ de las mujeres de 18 a 49 años. La Asociación Americana de Pediatría resalta el aumento del uso del condón en los adolescentes, paso de un 21\% en 1979 a 67\% en 1997 (20).

El uso de los anticonceptivos inyectables y los hormonales orales fueron del 22\% y $21.1 \%$ respectivamente; cifras que difieren a lo reportado por La Universidad El Bosque, donde los anovulatorios orales (44.8\%), anticonceptivos inyectables (18.8\%), el condón (31.4\%) fueron los más usados (21). En el estudio de La Universidad del Rosario, el porcentaje de uso de los métodos se distribuye así: anticonceptivos orales (15\%), coitus interruptus (14\%), anticonceptivos inyectables (7\%) y otros métodos (3\%) (9). La utilización de la anticoncepción la hacen los estudiantes por iniciativa propia en el $75.7 \%$, lo que demuestra el empoderamiento de los jóvenes frente a su salud sexual y reproductiva. Es importante tener en cuenta que el conocimiento no garantiza el uso de los métodos como lo reportan algunos estudios como el realizado en el Salvador donde se cerca del $90 \%$ de las adolescentes han oído hablar de los métodos anticonceptivos modernos, sin embargo, de las adolescentes con experiencia sexual, solamente una de cada 10 utilizó algún método anticonceptivo al momento de la primera relación sexual (11).

\section{Infecciones de transmisión sexual}

La gran mayoría de los estudiantes reportan tener conocimientos sobre las ITS, siendo la más conocida el VIH. En la ENDS 2.005 el 98 \% de las mujeres han oído hablar de esta enfermedad; en el Salvador un $77 \%$ de los jóvenes saben sobre la existencia del VIH. El nivel de conocimiento es menor en los grupos extremos de edad; es decir, entre las más jóvenes de 15 a 19 años y entre las de 40 a 49 ańos de edad. El 92.6\% de los estudiantes de La Universidad del Rosario manifiesta tener conocimientos adecuados sobre infecciones de transmisión sexual. En España se encontró que el 96\% de los jóvenes identifican las vías de transmisión del VIH y que un $15.7 \%$ aún tienen ideas erróneas sobre este aspecto; el 96,7\% identifican el preservativo como la medida más eficaz para la prevención del VIH (16). Cada minuto cinco personas de $15-24$ años de edad se infectan con el VIH; 33 millones de habitantes en el mundo han contraído la infección: de ellos, una tercera parte tiene entre 10 y 24 años, inciden África, con 1,7 millones y 700 mil en Asia y Pacífico (24).

Siendo la población estudiada sexualmente activa, un número muy bajo de ellos se han realizado la prueba del VIH (10\%), mientras que en España es del 39, $2 \%$, en Cuba el 18\% se han practicado la prueba. Los porcentajes más bajos de mujeres que se la han practicado corresponden a las más jóvenes ( 15 a 19 años de edad), en una población sexualmente activa es muy importante la realización de la prueba ya que los estudios reportan que ha aumentado la 
prevalencia del VIH en el grupo de 25 años lo que denota que la primo infección ocurre durante la adolescencia o juventud (15).

De los estudiantes que se realizaron la prueba del VIH el 37\% no recibieron asesoría pre y post prueba, una actividad muy importante para enfatizar sobre factores de riesgos, vulnerabilidad, protección y seguimiento, En México el 53\% de las mujeres que se practicaron la prueba recibido consejería antes del examen de laboratorio y $27 \%$ cuando recibió el resultado. El 92\% de las mujeres se conoció el resultado.

El 4.3\% de los estudiantes manifiesta haber tenido una ITS, cifra muy similar a la de los jóvenes de España, donde el 5,4\% de la población, que ha mantenido relaciones sexuales, refieren que le han diagnosticado alguna ITS a lo largo de su vida. En las mujeres las infecciones más frecuentes son las Trichomonas $(1,1 \%)$ Chlamydia (1\%), Herpes genital (0,8\%), pediculosis $(2,6 \%)$, Neisseria gonorrhoeae (1,2\%). Estos datos son más bajos que los encontrados en los jóvenes universitarios cubanos, donde se reporta que el 12\% se han contagiado con una ITS.

Otros reportes internacionales, informan criterios muy relevantes en relación con la repercusión de las ITS, en diferentes grupos poblacionales: 7 de cada 10 casos de se presenta entre 15-24 años de edad. Los adolescentes consideran que no corren riesgo porque creen, erróneamente, que las ITS sólo se transmiten entre las personas promiscuas o que adquieren "malos" comportamientos. Sin embargo, las adolescentes son más susceptibles adquirir ITS debido a la inmadurez del cuello uterino. Dicha vulnerabilidad también se encuentra asociada a la experiencia sexual temprana (24). Aunque la incidencia de ITS es baja, es muy preocupante que solo la mitad de las personas infectadas han recibido tratamiento; cifra similar a los datos obtenidos en La Universidad del Rosario, donde el 1\% de los encuestados ha tenido alguna ITS.

\section{Gestaciones}

El 10\% de la población ha presentado embarazos, casi la totalidad no son planeados y el $80 \%$ han terminado en aborto inducido. Comparando con Cuba, el porcentaje de embarazos es más bajo, pero el de abortos es muy similar. La perforación del útero y la esterilidad fueron las consecuencias más reconocidas y la minoría no identificó las posibles complicaciones. A pesar del conocimiento de los métodos anticonceptivos se sigue presentando una tasa muy alta de gestaciones no deseadas.

En Colombia, aunque en 1990 la tasa de fecundidad adolescente había descendido a 70 nacidos vivos por cada mil mujeres entre 15-19 años, en 1995 y en el 2000 aumenta nuevamente hasta alcanzar niveles cercanos a los observados hace treinta años, 85 por mil (25). En El Salvador el porcentaje de adolescentes sexualmente activas que para 1998 ya habían tenido al menos un embarazo fue del 80 $\%$ para las menores de 20 años y del $91 \%$ para las de 20 a 24 años de edad. Actualmente el promedio de edad es de 16 años para las menores de 20 ańos y de $17.9 \%$ para las de 20 a 24 años.

El embarazo en la adolescencia (menor de 20 años), es considerado por la OMS como un embarazo de riesgo, debido a las repercusiones que tiene sobre la salud de la madre y el producto de la concepción, las cuales contribuyen al aumento de la morbimortalidad materna y perinatal (26). Pero además del impacto en salud, el embarazo en la adolescente, sobre todo cuando no ha sido planeado, tiene repercusiones psicosociales y afecta los proyectos de vida de las jóvenes y genera problemas en su entorno familiar, social y contribuye en gran medida a la deserción escolar (27).

\section{Prevención de cáncer en mujeres}

Un alto porcentaje de la población presenta antecedentes de cáncer en la familia, factor predisponente a tener en cuenta en la planeación y ejecución de un programa de SSR universitario. Solo un poco más de la mitad de las mujeres se realizan el examen de seno, cifra muy similar a lo reportado en la ENDS. En cuanto a la regularidad del examen, casi la mitad reporta que se lo realiza mensualmente, siendo una cifra superior a lo reportado por la ENDS, donde el $25 \%$ de las mujeres lo realizan cada mes y el $3 \%$ cada 6 meses. El 80\% de las mujeres sabe qué es el auto examen de seno. El conocimiento es mayor en la zona urbana, aumenta con el nivel educativo (8).

La mitad de las estudiantes se realizan el examen en cualquier momento del ciclo menstrual, un poco más bajo que lo encontrado en Colombia según la ENDS donde es del 70\%. Solamente el 2\% lo hace 
regularmente. Frente a las recomendaciones médicas, se confronta un desconocimiento de las mujeres sobre el mejor momento para realizarse el auto examen. Un porcentaje muy bajo de estudiantes han presentado anormalidades en el seno (8).

La edad de la menarquia es un factor a tener en cuenta como factor predisponente para el desarrollo de cáncer de seno y la gran mayoría de las estudiantes la tuvieron antes de los 13 años. Para ser una población sexualmente activa es muy baja la frecuencia de realización de la citología. La citología es realizada anualmente por el $66 \%$ de las estudiantes, cifra superior a lo encontrado en la población colombiana donde es del 48\%; el 13\% más de una vez al año, el $4 \%$ cada dos ańos; el $22 \%$ rara vez y el $13 \%$ una vez. Cabe destacar que en la población estudiantil un $36,8 \%$ se realiza la citología sin ninguna regularidad perdiendo así su valor de prevención y tratamiento oportuno. Un $86 \%$ de las estudiantes conoció el resultado de la citología, cifra más baja a lo reportado por ENDS que fue del 92\% (8).

En cuanto al resultado de la citología, el 8,6\% fue anormal, tendencia muy similar a la nacional, donde es uno de cada diez. El 8\% fue a nueva consulta para el tratamiento. Los resultados anormales son más altos para las menores de 35 ańos, pero no varían mucho de acuerdo con la educación de las mujeres, el rango de variación está entre $7 \%$ con educación superior y $10 \%$ con secundaria. Según la ENDS, el $4 \%$ no reclamaron los resultados de la última citología vaginal; al 38\% la institución no se las entregó y al $19 \%$ no les interesó reclamar los resultados. El 3\% tuvo miedo que le dijeran que tenía cáncer (8).

\section{Asesoría en salud sexual y reproductiva}

Los autores de este estudio quisieron involucrar a los jóvenes en la búsqueda de soluciones y propuestas a desarrollar en un futuro en la universidad, que respondieran a sus necesidades, para lo cual se indagó sobre la viabilidad de implementar un programa de salud sexual y reproductiva. Los medios para preferidos para tener acceso a este programa fueron la consulta y asesoría individualizada y la pagina web. Los temas de mayor interés fueron la prevención de las infecciones de transmisión sexual y la prevención de embarazos.
Para finalizar, el desarrollo de esta investigación determinó las conductas de salud y los factores de riesgo dentro de población universitaria, que comparten características con otras comunidades universitarias del país. Estos resultados permiten orientar y proponer acciones concretas que respondan a sus necesidades.

Teniendo en cuenta los factores protectores y de riesgo detectados en la población universitaria, nuestras recomendaciones son: difundir los resultados de este estudio dentro de la comunidad científica y educativa de la universidad y fuera de ella, para generar conocimientos sobre factores protectores y conductas de salud en los jóvenes, que sirvan para orientar acciones específicas en los campos de salud y educación con el objetivo de promover el ejercicio de una sexualidad sana y libre de riesgos. Promover en la Universidad el programa de salud sexual y reproductiva con asesorías individualizadas y a través de medios virtuales, que requiere el concurso de las diferentes facultades, del servicio médico de la Universidad.

\section{Referencias}

1. Ministerio de la Protección Social. Salud Sexual Colombia. Política Nacional de Salud Sexual y Reproductiva (SSR) de 2003; Febrero de 2003.

2. Objetivos de desarrollo del Milenio Informe de 2005.

3. República de Colombia, Ministerio de la Protección Social. Resolución Número 00412 de 2000.

4. Alcaldía Mayor Santa Fe de Bogotá, Secretaría Distrital de Salud. Lineamientos generales en salud sexual y reproductiva para Santa Fe de Bogotá, D.C.; 2006.

5. Organización Panamericana de la Salud. Fecundidad en la adolescencia. Causas. Riesgos y opciones. Cuaderno Técnico No 12. Washington; 1998.

6. Castro EM. Crecer en la adolescencia: Retos actuales de la Educación Superior y una propuesta para la población. Sexología y sociedad. 1996;4:2-6.

7. Victoria S. Salud Sexual y Reproductiva de las Adolescentes en Colombia. Informe para el FNUAP. Bogotá, Colombia.2006;8-10.

8. Asociación pro bienestar de la Familia Colombiana PROFAMILIA. Salud sexual y reproductiva en Colombia Encuesta Nacional de Demografía y Salud 2005.

9. Ruiz AM, Latorre C, Beltran J, Ruiz J, Vélez A. Conocimientos, actitudes y prácticas en salud sexual y reproductiva en una población universitaria. Rev Sogia. 2005;12:86-93.

10. Posada C. Realidades y Retos del Embarazo Adolescente. Medellín: CERFAMI, 2000.

11. Centeno H, Cáceres R. La salud sexual y reproductiva de las jóvenes de 15 a 24 años El Salvador, un reto para las políticas de salud. Población y Salud en Meso América [revista en internet]*2005 enerojunio. [acceso 26 de mayo de 2009]; 2(2). Disponible en: http://ccp. ucr.ac.cr/revista/volumenes/2/2-2/2-2-1/ 
12. Organización Mundial de la Salud. Documento básico para las discusiones técnicas sobre la salud de los jóvenes. In: Asamblea mundial de la salud; 1989; Ginebra, 1989.

13. Centers for Disease Control and Prevention. Youth Risk Behavior Surveillance: National College Health Risk Behavior Survey - United States, 1995. MMWR CDC Surveill Summ 1997;46:1-56.

14. González L, González N, Fernández M, Navarro A, Linares X. Salud Sexual y Reproductiva en Estudiantes Universitarios. ¿Modo y Estilo de Vida Saludables?, Un diagnóstico. Rev Hum Med [revista en internet] 2003 Ene - Abr. [acceso 26 de mayo de 2009]; 3 (1). Disponible en: http://bvs.sld.cu/revistas/revistahm/numeros/2003/ n7/body/hmc010103.htm

15. Academia Nacional de Medicina de México, A.C. El modelo universitario de salud sexual y reproductiva: ¿Quo Vadis México?. Gac Méd Méx Vol. 139, Suplemento No. 4, 2003MG. Disponible en: http:// www.medigraphic.com/pdfs/gaceta/gm-2003/gms034f.pdf.

16. INE (2004). Salud y hábitos sexuales. Las conductas sexuales desde la perspectiva del SIDA. Boletín Informativo del Instituto Nacional de Estadística 2004.Recuperado el 10 de marzo de 2005. Disponible en: http://www.ine.es/revistas/cifraine/cifine_sida0704.pdf.

17. Tang CS, Lai FD, Chung TKH. Assessment of sexual functioning for chinese college students. Archives of Sexual Behavior. 1997;26:79-90.

18. Maddaleno M. Atención de salud del adolescente: un modelo en el nivel primario de atención en un sector urbano marginal de Santiago de Chile. En: La Salud del adolescente en Chile: Corporación de promoción Universitaria; 1988.

19. La salud sexual y reproductiva de las jóvenes de 15 a 24 años El Salvador, un reto para las políticas de salud. Helen Larissa Centeno Monge1 Rhina Cáceres Rodas2 2005.
20 American Academy of Pediatrics Committee on Adolescence. Condom use by Adolescents. Pediatrics 2001;107:1463-1469.

21. Arteaga M, Daza S, Gómez N, Raga N. Asociación entre conocimientos y uso de métodos anticonceptivos en estudiantes de Enfermería y Medicina de la Universidad El Bosque. Revista Colombiana de Enfermeria. 2006;1:65-73.

22. Aggarwal O, Sharma AK, Chabra P. Study in sexuality of medical college students in India. Journal of Adolescence Health. 2000;26:226-229.

23. Fridman L, Ferguson B. Enfoques de la OMS sobre la salud de los adolescentes. Publicaciones de la Organización Mundial de la Salud, Ginebra, 1995.

24. Guerrero N. El valor de la educación sexual. Sexología y Sociedad. $1995 ; 2: 14-15$.

25. Flórez C, Vargas E, Henao J, González C. Fecundidad Adolescente en Colombia: Incidencia, Tendencias Y Determinantes. Un Enfoque De Historia De Vida. Documento CEDE No 31. 2004. Bogotá: Universidad de los Andes.

26. Programming for Adolescent Health and Development. Report of a WHO/UNFPA/UNICEF Study Group on Programming for Adolescent Health. WHO Technical Report Series, Geneva, 1999.

27. Pérez G, Beltrán F, Benet C, García E, Fernández E, Garza J. La Salud Sexual y Reproductiva de los y las Adolescentes: Una Estrategia Preventiva. Publicaciones de la Secretaría de Salud. Ediciones Buena Tinta SA de CV, México, D.F., 2000. 